\title{
Structure-Function Correlation in Novel Nanomedicines for RNA Delivery
}

\author{
Sara S. Nogueira ${ }^{1}$, Jorge Moreno ${ }^{1,2}$, Heinrich Haas ${ }^{1}$, Kerstin Reuter $^{1}$, Stephanie Erbar $^{1}$, Peter Languth ${ }^{2}$, \\ Ugur Sahin² \\ ${ }^{1}$ BioNTech RNA Pharmaceuticals \\ An der Goldgrube, Mainz, Germany \\ sara.nogueira@biontech.de \\ ${ }^{2}$ Johannes Gutenberg-Universität \\ Mainz, 55099 Mainz, Germany
}

\section{Extended Abstract}

Messenger RNA (mRNA)-based nanomedicines constitute a new class of pharmaceutical products, with a variety of potential applications, ranging from tumour immunotherapy to protein substitution. For patient administration, mRNA can be formulated in different types of nanoparticle vehicles, in order to protect the mRNA from degradation and facilitate uptake resulting in expression at the target site. BioNTech has brought the first intravenously injectable mRNA nanoparticle product for cancer immunotherapy into clinical trials, which consist of a lipoplex formulation obtainable from cationic liposomes $[1,2]$.

Lipid nanoparticles (LNPs) are another type of delivery vehicle and have been successfully used in the past, for example, to deliver siRNA to the liver [3]. Recently, LNPs have been also been used as carriers for mRNA, for induction potent CD8 $\mathrm{T}$ cell immune response [4], showing that LNPs can be versatile delivery systems for RNA in diverse therapeutic settings.

Typically, LNPs are comprised of an ionizable lipid, one or several helper lipids and a polyethylene glycol (PEG). The ionizable lipid, which is positively charged at low $\mathrm{pH}$ and neutral at high $\mathrm{pH}$, is selected to allow high RNA encapsulation and efficacy and to facilitate endosomal escape. The activity of the mRNA LNP formulations strongly depends on the type of ionisable lipid, on the lipid composition, the lipid to RNA ratio, and several other molecular and structural parameters. Furthermore, the activity may vary also for different application routes (e.g., intravenous, intramuscular, subcutaneous), or if therapeutic approaches are intended. So far, there is no clear common understanding on the correlation between the molecular parameters of the LNPs and the biological activity. Therefore, in our study we have systematically studied certain molecular and formulation parameters of mRNA loaded LNPs, in order to correlate them with the biological function. In particular we have thoroughly investigated physicochemical characteristics (internal organization, fluidity, size) and the structure of the LNPs (in particular by small angle x-ray scattering) and determined the biological activity in vitro and in vivo. The structural and functional coherencies in the LNPs were compared in those of lipoplexes, and the effects of selected lipids were highlighted. Such understanding of the molecular basis of delivery complexes will help to identify criteria for the development of improved mRNA delivery vehicles for clinical development.

Keywords: RNA, Lipid Nanoparticles, Drug Delivery, Structure-function correlation

\section{References}

[1] L. M. Kranz, "Systemic RNA delivery to dendritic cells exploits antiviral defence for cancer immunotherapy," Nature, vol. 534, pp. 396-401, 2016.

[2] S. Grabbe, "Translating nanoparticulate-personalized cancer vaccines into clinical applications: Case study with RNA-lipoplexes for the treatment of melanoma," Nanomedicine, vol. 11, no. 20, pp. 2723-2734, 2016.

[3] S. Chen, "Development of lipid nanoparticle formulations of siRNA for hepatocyte gene silencing following subcutaneous administration," J Control Release, vol. 196, pp. 106-112, 2014. 
[4] M. A. Oberli, "Lipid Nanoparticle-Assisted mRNA Delivery for Potent Cancer Immunotherapy," Nano Letters, 2016. 